\title{
Erratum to: Les troubles moteurs osophagiens au cours de la sclérodermie systémique
}

\section{Erratum to: Esophageal motility abnormalities in systemic sclerosis}

K. Saké • N. Amrani - I. Serraj • M. Nya - R. Razine - N. Amrani

C) Springer-Verlag France 2013

Erratum to : Acta Endosc. DOI 10.1007/s10190-012-0290-4

Les auteurs sont K. Saké, L. Amrani, I. Serraj, M. Nya, R. Razine, N. Amrani.

Et l'auteur correspondant de cet article est N. Amrani. 